\title{
Separability criterion for bipartite states and its generalization to multipartite systems
}

\author{
Jie-Hui Huang ${ }^{1, *}$, Li-Yun $\mathrm{Hu}^{1}$, Lei $\mathrm{Wang}^{2}$, and Shi-Yao Zhu ${ }^{3}$ \\ ${ }^{1}$ College of Physics and Communication Electronics, \\ Jiangxi Normal University, Nanchang 330022, People's Republic of China \\ ${ }^{2}$ College of Physics, Jilin University, Changchun 130021, People's Republic of China \\ ${ }^{3}$ Beijing Computational Science Research Center, Beijing 100084, People's Republic of China
}

\begin{abstract}
A group of symmetric operators are introduced to carry out the separability criterion for bipartite and multipartite quantum states. Every symmetric operator, represented by a symmetric matrix with only two nonzero elements, and their arbitrary linear combinations are found to be entanglement witnesses. By using these symmetric operators, Wootters' separability criterion for two-qubit states can be generalized to bipartite and multipartite systems in arbitrary dimensions.
\end{abstract}

PACS numbers: 03.67.Mn, 03.65.Fd

Quantum entanglement, introduced in the early days of quantum theory [1], not only plays a vital role in differentiating quantum mechanics from classical mechanics, but also acts as key resource in quantum information processing. Thus the verification of quantum entanglement is fundamentally important in quantum information science. Since the mathematical description on quantum entanglement was first introduced by Werner in 1989 [2], many entanglement measures have been proposed, such as distillable entanglement, entanglement cost [3] , relative entropy of entanglement [4], Schmidt number [5], concurrence [6, 7], negativity [8], and so on [9]. Recently, the verification of multipartite entanglement and relevant issues are attracting more and more attention [10, 11] owing to the booming interest on quantum many-body systems [12].

The convexity requirement for quantum entanglement, which is in accord with the fact local operations with classical communication (LOCC) can not create or increase quantum entanglement [3], makes it very hard to verify the entanglement of mixed quantum states. Historically, positive partial transpose (PPT) criterion, proposed by Peres in 1996 [13], provides a necessary and sufficient condition for verifying the separability of $2 \otimes 2$ and $2 \otimes 3$ bipartite systems [14]. But for higher dimensional systems, how to distinguish entangled states from separable ones is still a challenging question. In this Letter, we introduce a group of symmetric operators to answer this question. It is shown that all these symmetric operators and their arbitrary linear combinations are entanglement witnesses. We use these symmetric operators to generalize Wootters' concurrence [6, 7] to high dimensional bipartite [15, 16] and multipartite systems [17]. More importantly, based on these symmetric operators the separability criterion for bipartite and multipartite states is presented in arbitrary dimensions, which is a generalization of Wootters' separability criterion from the two-qubit case to a general case.

In the simplest two-qubit system, a pure state $|\psi\rangle=\sum_{i=1}^{2} \sum_{j=1}^{2} a_{i j} \mid i_{1} j_{2}>$, where the subscripts "1" and "2" in the state vectors denote the two subsystems, is separable when it is a tensor product of a pure state in subsystem " 1 " and a pure state in subsystem " 2 ", thus the four coefficients $a_{i j}(i, j \in\{1,2\})$ have to satisfy the condition $a_{11} a_{22}=a_{12} a_{21}$. In Ref.[7], Wootters introduced a "spin-flip" operator $\sigma_{y}$ (Pauli matrix) in the standard basis to verify the separability of a two-qubit pure state. That is, a two-qubit pure state $|\psi\rangle$ is separable iff $\left\langle\psi^{*}\left|\sigma_{y} \otimes \sigma_{y}\right| \psi\right\rangle=0$. In the following, we call the state vector $\mid \psi>$ and its complex conjugate $\mid \psi^{*}>$ orthogonal with respect to the operator $O$, or simply $O$-orthogonal, if they satisfy the relation $\left\langle\psi^{*}|O| \psi\right\rangle=0$. Wootters' separability criterion for a two-qubit pure state can then be stated as, it should be $O$-orthogonal to its complex conjugate with the operator $O=\sigma_{y} \otimes \sigma_{y}$. Here the antisymmetry of the "spin-flip" operator $\sigma_{y}$, i.e. $\sigma_{y}^{T}=-\sigma_{y}$ (the superscript "T" stands for matrix transpose hereafter), plays the essential role in verifying the separability of a two-qubit state. This $O$-orthogonal relation between a separable two-qubit pure state and its complex conjugate is violated by all entangled two-qubit states.

Now we consider a general bipartite case, with the two subsystems having dimensions $D_{1}$ and $D_{2}$, respectively. If a bipartite pure state $|\psi\rangle=\sum_{i=1}^{D_{1}} \sum_{j=1}^{D_{2}} a_{i j} \mid i_{1} j_{2}>$ is separable, the four coefficients $a_{i j}, a_{i j^{\prime}}, a_{i^{\prime} j}$, and $a_{i^{\prime} j^{\prime}}$ with $i, i^{\prime} \in\left\{1,2, \cdots, D_{1}\right\}$ and $j, j^{\prime} \in\left\{1,2, \ldots, D_{2}\right\}$, have to satisfy the condition $a_{i j} a_{i^{\prime} j^{\prime}}=a_{i j^{\prime}} a_{i^{\prime} j}$, so that this bipartite pure state can be formulated as a tensor product. This condition, similar to the above two-qubit case, is equivalent to the following $O^{\left(i, i^{\prime}: j, j^{\prime}\right)}$-orthogonal relation,

$$
<\psi^{*}\left|O^{\left(i, i^{\prime}: j, j^{\prime}\right)}\right| \psi>=0
$$

with

$$
O^{\left(i, i^{\prime}: j, j^{\prime}\right)}=\left[\sigma_{1}^{\left(i, i^{\prime}\right)}-\left(\sigma_{1}^{\left(i, i^{\prime}\right)}\right)^{T}\right] \otimes\left[\sigma_{2}^{\left(j, j^{\prime}\right)}-\left(\sigma_{2}^{\left(j, j^{\prime}\right)}\right)^{T}\right] .
$$

Here the operator $\sigma_{1}^{\left(i, i^{\prime}\right)}\left(\sigma_{2}^{\left(j, j^{\prime}\right)}\right)$ in the subsystem "1" ("2") has only one nonzero element "1" located at row $i(j)$ and column $i^{\prime}\left(j^{\prime}\right)$ in the standard basis, no matter what dimensions it has. The two antisymmetric (or skew-symmetric) 
operators, $\left[\sigma_{1}^{\left(i, i^{\prime}\right)}-\left(\sigma_{1}^{\left(i, i^{\prime}\right)}\right)^{T}\right]$ and $\left[\sigma_{2}^{\left(j, j^{\prime}\right)}-\left(\sigma_{2}^{\left(j, j^{\prime}\right)}\right)^{T}\right]$, which play the same role as the antisymmetric operator $\sigma_{y}$ in the two-qubit case, establish a relation among the four coefficients $a_{i j}, a_{i j^{\prime}}, a_{i^{\prime} j}$, and $a_{i^{\prime} j^{\prime}}$.

Since the superscript numbers $i, i^{\prime} \in\left\{1,2, \cdots, D_{1}\right\}$ and $j, j^{\prime} \in\left\{1,2, \ldots, D_{2}\right\}$ in the above Eq.(1) can be chosen arbitrarily, the $O^{\left(i, i^{\prime}: j, j^{\prime}\right)}$-orthogonal relation (1a) for a separable bipartite pure state, holds valid for all possible operators $O^{\left(i, i^{\prime}: j, j^{\prime}\right)}$ in the form (1b). Owing to the linear dependence of the relation (1a) on the operators $O^{\left(i, i^{\prime}: j, j^{\prime}\right)}$, we even can replace the operators $\sigma_{1}^{\left(i, i^{\prime}\right)}$ and $\sigma_{2}^{\left(j, j^{\prime}\right)}$ by a random matrix $\sigma_{1}^{(\mathrm{rdm})}$ in subsystem "1" and a random matrix $\sigma_{2}^{(\mathrm{rdm})}$ in subsystem "2" to construct,

$$
O^{(\mathrm{Semi}-\mathrm{rdm})}=\left[\sigma_{1}^{(\mathrm{rdm})}-\left(\sigma_{1}^{(\mathrm{rdm})}\right)^{T}\right] \otimes\left[\sigma_{2}^{(\mathrm{rdm})}-\left(\sigma_{2}^{(\mathrm{rdm})}\right)^{T}\right],
$$

and the $O^{\text {(Semi-rdm) }}$-orthogonal relation, $<\psi^{*}\left|O^{(\text {Semi-rdm) }}\right| \psi>=0$, still holds true if only the pure state $\mid \psi>$ is separable. Thus we can conclude that a bipartite pure state in arbitrary dimensions is separable iff it and its complex conjugate are orthogonal with respect to all symmetric operators formulated as a tensor product of two antisymmetric operators in the two subsystems. To verify the separability of a given bipartite pure state, we only need to pick out a finite number of linearly independent symmetric operators and check the above $O^{\text {(Semi-rdm) }}$-orthogonal relation. A violation of the $O^{\text {(Semi-rdm) }}$-orthogonal relation by any operator $O^{\text {(Semi-rdm) }}$ is enough to declare the existence of quantum entanglement.

Now we use a complete set of linearly independent symmetric operators $O^{\left(i, i^{\prime}: j, j^{\prime}\right)}$, which are directly constructed by $\frac{1}{2} D_{1}\left(D_{1}-1\right)$ linearly independent antisymmetric operators $\left[\sigma_{1}^{\left(i, i^{\prime}\right)}-\left(\sigma_{1}^{\left(i, i^{\prime}\right)}\right)^{T}\right]$ in subsystem "1" and $\frac{1}{2} D_{2}\left(D_{2}-1\right)$ linearly independent antisymmetric operators $\left[\sigma_{2}^{\left(j, j^{\prime}\right)}-\left(\sigma_{2}^{\left(j, j^{\prime}\right)}\right)^{T}\right]$ in subsystem "2", to define the entanglement measure of a bipartite pure state $|\psi\rangle$,

$$
C^{(2)}(|\psi\rangle)=\sqrt{\sum_{i=1}^{D_{1}-1} \sum_{i^{\prime}=i+1}^{D_{1}} \sum_{j=1}^{D_{2}-1} \sum_{j^{\prime}=j+1}^{D_{2}}\left|<\psi^{*}\right|\left[\sigma_{1}^{\left(i, i^{\prime}\right)}-\left(\sigma_{1}^{\left(i, i^{\prime}\right)}\right)^{T}\right] \otimes\left[\sigma_{2}^{\left(j, j^{\prime}\right)}-\left(\sigma_{2}^{\left(j, j^{\prime}\right)}\right)^{T}\right]|\psi>|^{2}},
$$

where the superscript "(2)" denotes the bipartite system. This operator-based entanglement measure for bipartite pure states, which we call $O$ concurrence in the following, turns back to Wootters' concurrence for two-qubit systems, and satisfies the basic requirements for a good entanglement measure. For example, (i) it presents zero result for all tensor product states, and positive results for entangled states; (ii) it remains invariant under all local unitary transformations, and so on. In fact, this $O$ concurrence is the same as measured in the following way,

$$
C^{(2)}(\mid \psi>)=\sqrt{2-\operatorname{tr}\left(\rho_{1}^{2}\right)-\operatorname{tr}\left(\rho_{2}^{2}\right)}
$$

with $\rho_{1}\left(\rho_{2}\right)$ being the reduced density matrix of subsystem "1" ("2"), and " $\operatorname{tr}()$ " denoting matrix trace. Since the equality $\operatorname{tr}\left(\rho_{1}^{2}\right)=\operatorname{tr}\left(\rho_{2}^{2}\right)$ holds for bipartite pure states, our entanglement measure is equivalent to the entanglement measure proposed in Ref. [16], which is called I concurrence owing to its connection to the universal inverter. It is very interesting that two measures present the same result for the bipartite entanglement, though they are defined in two different ways and based on different ideas.

This $O$ concurrence can also be extended by convex roof to measure the entanglement of mixed bipartite states. At present, we only concern the separability condition of mixed bipartite states, rather than the evaluation of their entanglement. According to Werner's criterion [2], a mixed bipartite state $\rho$ is separable only when it can be written as a mixture of separable pure states,

$$
\rho=\sum_{i, j} P_{i j}\left|\psi_{1}^{i}><\psi_{1}^{i}\right| \otimes\left|\psi_{2}^{j}><\psi_{2}^{j}\right|,
$$

with the probabilities $P_{i j} \geq 0$ and $\sum_{i, j} P_{i j}=1$. However, every mixed quantum state has countless types of purestate decomposition. Given a density matrix, it is usually very difficult to prove the existence or nonexistence of a decomposition composed of product states. In other words, we can easily construct a separable mixed quantum state by mixing a group of separable states in the above way (5). However, it is very difficult to recover this group of separable states, provided only the density matrix.

We begin our investigation on the separability of a mixed $D_{1} \otimes D_{2}$ state with a Hermitian matrix $\sqrt{\rho}$, which is the square root of the density matrix $\rho$. Every pure-state decomposition of the density matrix $\rho$ is connected to a $D_{12} \times M\left(D_{12}=D_{1} \times D_{2} \leq M\right)$ right-unitary transformation $U$ through the relation, $W=\sqrt{\rho} U$. Each column vector of the matrix $W$, the $i$ th column vector denoted as $\left|W_{i}\right\rangle$ hereafter, is a "subnormalized" pure state in the $U$-decomposition, and the original density matrix can then be written as $\rho=\sum_{i=1}^{M}\left|W_{i}><W_{i}\right|$. Now we suppose 
that the density matrix $\rho$ represents a separable mixed state, which means there exists at least one $U$-decomposition so that all pure states in this decomposition are tensor product states. That is to say, every column vector $\mid W_{i}>$ is $O^{\text {(Semi-rdm) }}$-orthogonal to its complex conjugate, with the operator $O^{\text {(Semi-rdm) }}$ defined in Eq.(2).

Since all column vectors $\mid W_{i}>$ satisfy the $O^{\text {(Semi-rdm) }}$-orthogonal relation, $\left.<W_{i}^{*}\left|O^{(\text {Semi-rdm })}\right| W_{i}\right\rangle=0$, for a particular $U$-decomposition of the separable mixed state $\rho$, the symmetric matrix $W^{T} O^{(\text {Semi-rdm) }} W$ is a hollow matrix whose diagonal elements are all equal to zero. Equivalently speaking, a mixed bipartite state $\rho$ is separable, iff there exists a right-unitary transformation $U$, so that the symmetric matrix $U^{T} S U$, with

$$
S=(\sqrt{\rho})^{T} O^{(\text {Semi-rdm })} \sqrt{\rho},
$$

is a hollow matrix for all operators $O^{\text {(Semi-rdm) }}$ in the form (2). Here we emphasize the above symmetric matrix $S$ depends only on the given density matrix and an operator constructed by two random matrices. Now a new question arises, given a symmetric matrix $S$, whether there exists such a right-unitary transformation $U$ so that $U^{T} S U$ is a hollow matrix?

We can find out the answer by using Wootters' method in Ref.[7]. A symmetric matrix $S$ can be transformed to a hollow matrix in the way of $U^{T} S U$, only when its maximal singular value is no larger than the sum of the rest singular values. Supposing $\left\{\lambda_{i}\right\}$ are singular values of the symmetric matrix $S$ in decreasing order, the above statement is equivalent to the following condition,

$$
\lambda_{1} \leq \sum_{i=2} \lambda_{i}
$$

On the contrary, if the above condition (7) is not satisfied for even one particular operator in the form (2), e.g. $O^{\text {(particular) }}=\sum_{i=1}^{D_{1}-1} \sum_{i^{\prime}=i+1}^{D_{1}} \sum_{j=1}^{D_{2}-1} \sum_{j^{\prime}=j+1}^{D_{2}} c_{i, i^{\prime}: j, j^{\prime}} O^{\left(i, i^{\prime}: j, j^{\prime}\right)}$, the mixed state $\rho$ must be entangled. Please see Eq. (1b) for the definition of the operator $O^{\left(i, i^{\prime}: j, j^{\prime}\right)}$. Without loss of generality, we assume the first coefficients $c_{12: 12}$ has the maximal modulus among all the complex coefficients $\left\{c_{i, i^{\prime}: j, j^{\prime}}\right\}$. In this case, the average $O$ concurrence of the pure states, i.e. column vectors $\left\{\mid W_{k}>\right\}$, in an arbitrary $U$-decomposition, $C_{U}^{(2)}(\rho)=\sum_{k=1}^{M} C^{(2)}\left(\mid W_{k}>\right)$, is larger than zero, because $C_{U}^{(2)}(\rho) \geq \frac{1}{\sqrt{\left|c_{12: 12}\right|}}\left(\lambda_{1}-\sum_{j=2}^{D_{12}} \lambda_{j}\right)$. So, given a mixed bipartite state $\rho$, if the condition (77) is violated by even one operator in the form (2), this mixed state is an entangled state. One of main conclusions in this Letters can now be concluded, a mixed bipartite state in arbitrary dimensions is separable only when the condition (7) for the singular values of the symmetric matrix (66) is generally true for all operators $O^{(\text {Semi-rdm) }}$ in the form (2). This is a necessary and sufficient condition. On the one hand, if the condition (7) is valid for all operators $O^{\text {(Semi-rdm) }}$ in the form (2), there must exist a unitary transformation $U$ independent on the operator $O^{\text {(Semi-rdm) }}$ (because it is constructed by a random matrix $\sigma_{1}^{(\mathrm{rdm})}$ in subsystem " 1 ", and a random matrix $\sigma_{2}^{(\mathrm{rdm})}$ in subsystem "2" ), to satisfy all $O^{\text {(Semi-rdm) }}$-orthogonal relations in the corresponding decomposition, and such a bipartite state is separable; On the other hand, any violation of this condition, just as we already shown, is enough to declare the existence of quantum entanglement. In other words, every operator $O^{\text {(Semi-rdm) }}$ in the form (2) is a witness [19] of bipartite entanglement under the violation of the condition (7).

The singular values $\left\{\lambda_{i}\right\}$ of the symmetric matrix $S$ (6) are equal to the square root of the eigenvalues of the matrix $S^{\dagger} S$ [18], and as well the square root of the eigenvalues of the following matrix,

$$
\rho\left[O^{(\text {Semi-rdm })}\right]^{\dagger} \rho^{*} O^{(\text {Semi-rdm })},
$$

which is very similar to the form used in the concurrence paper [7], and can be considered as its generalization in high dimensional systems.

At present, we can not verify the separability of a bipartite state in high dimensions through finite tests on the condition (7), unless it is a pure state. But we here provided an efficient way for verifying the entanglement of a bipartite state, because any operator $O^{(\text {Semi-rdm) }}$ in the form (2) is an entanglement witness under the violation of the condition (77). Our numerical results show that the larger entanglement a bipartite state contains, the fewer tests it usually requires to find a violation by randomly generating the matrices $\sigma_{1}^{(\mathrm{rdm})}$ and $\sigma_{2}^{(\mathrm{rdm})}$ in the form (2).

Now we come to the multipartite case. A multipartite pure state is said to be entangled only when it cannot be written as a tensor product of the states in the subsystems. This definition does not distinguish between "truly multipartite" entanglement and low-partite entanglement [20]. For example, both the Greenberger-Horne-Zeilinger state and the $W$ state in the three-qubit system are tripartite entangled states according to the above definition, but only the former one has nonzero 3-tangle for "truly tripartite" entanglement [10]. Our following discussion on the separability criterion of multipartite states is based on this definition of entanglement, no matter what kind of entanglement, "truly multipartite" entanglement or low-partite entanglement, is contained. 
For a separable multipartite pure state,

$$
|\psi>=| \psi_{1}>\otimes\left|\psi_{2}>\otimes \cdots \otimes\right| \psi_{N}>
$$

each subsystem is separable with the rest subsystems as a whole. The inverse situation is also true. Among a multipartite system, if every subsystem is separable with the rest subsystems as a whole, this multipartite pure state is separable. That is to say, multipartite entanglement can be featured by bipartite entanglement. Thus the entanglement degree of a multipartite pure state can be quantified by $C^{(N)}(\mid \psi>)=\sqrt{\frac{1}{2} \sum_{k=1}^{N}\left|C^{(2)}\left(\mid \psi_{k \bar{k}}>\right)\right|^{2}}$, where the superscript " $(N)$ " denotes $N$-partite system, $C^{(2)}$ is the bipartite entanglement defined in Eq.(3), and $\mid \psi_{k \bar{k}}>$ is the bipartite version of the quantum state $\mid \psi>$ in Eq. (9) by considering $(N-1)$ subsystems, excluding the $k$ th one, as a whole. Although some other bipartite entanglement, e.g. $C^{(2)}\left(\left|\psi_{(12)(\overline{12})}\right\rangle\right)$, where the first part $(12)$ is composed of the two subsystems " 1 " and " 2 ", and the other part $(\overline{12})$ is composed of the rest $(N-2)$ subsystems, can also be included to define other types of multipartite entanglement, for example in Ref. [21], the participation of these terms only affects the evaluation of entanglement, but does not change the separability criterion. It means we can measure the multipartite entanglement in a simpler way, where every involved bipartite entanglement is associated with one single subsystem and the rest as a whole. The above entanglement measure for multipartite states can also be described in terms of operators,

$$
C^{(N)}(|\psi\rangle)=\sqrt{\left.\frac{1}{8} \sum_{k=1}^{N} \sum_{i, i^{\prime}=1}^{D_{1}} \cdots \sum_{j, j^{\prime}=1}^{D_{N}}\left|<\psi^{*}\right| O_{k \bar{k}}^{\left(i, i^{\prime}: \cdots: j, j^{\prime}\right)}|\psi\rangle\right|^{2}}
$$

where the symmetric operator,

$$
O_{k \bar{k}}^{\left(i, i^{\prime}: \cdots: j, j^{\prime}\right)}=\sigma_{1}^{\left(i, i^{\prime}\right)} \otimes \cdots \otimes\left[\sigma_{k}^{\left(m, m^{\prime}\right)}-\left(\sigma_{k}^{\left(m, m^{\prime}\right)}\right)^{T}\right] \otimes \cdots \otimes \sigma_{N}^{\left(j, j^{\prime}\right)}+T . c .,
$$

is associated the bipartite system composed of the $k$ th subsystem and all the other subsystems as a whole, $D_{k}$ is the dimension degree of the $k$ th subsystem and T.c. means matrix transpose. This is generalized $O$ concurrence for multipartite pure states, which, inherited from bipartite entanglement, remains invariant under local unitary transformations. Under this measure, the maximally-entangled $D$-dimensional $N$-partite Greenberger-Horne-Zeilinger state $\left|G H Z^{(N)}>=\frac{1}{\sqrt{D}} \sum_{i=1}^{D}\right| i_{1} i_{2} \cdots i_{N}>$ has entanglement $\sqrt{N\left(1-\frac{1}{D}\right)}$, and the generalized $N$-qubit $W$ state $\mid W^{(N)}>=\frac{1}{\sqrt{N}}(|100 \cdots>+| 010 \cdots>+\cdots+\mid 00 \cdots 1>)$ has entanglement $\sqrt{2\left(1-\frac{1}{N}\right)}$. Similar to the bipartite case, the entanglement measure (10) has an equivalent but much simpler version,

$$
C^{(N)}(\mid \psi>)=\sqrt{N-\sum_{k=1}^{N} \operatorname{tr}\left(\rho_{k}^{2}\right)}
$$

where $\rho_{k}$ stands for the reduced density matrix of the $k$ th subsystem. This measure is a generalization of the $I$ concurrence [16] for multipartite systems.

Since the present entanglement measure for multipartite systems is based on the bipartite entanglement between single subsystems and the rest as a whole, we can directly generalize some conclusions from bipartite case to the present multipartite case. For example, a multipartite pure state is separable only when it is orthogonal to its complex conjugate with respect to all operators in the form (11), and also their extended version,

$$
O_{k \bar{k}}^{(\mathrm{Semi}-\mathrm{rdm})}=\sigma_{1}^{(\mathrm{rdm})} \otimes \cdots \otimes\left[\sigma_{k}^{(\mathrm{rdm})}-\left(\sigma_{k}^{(\mathrm{rdm})}\right)^{T}\right] \otimes \cdots \otimes \sigma_{N}^{(\mathrm{rdm})}+T . c .,
$$

where $\sigma_{k}^{(\mathrm{rdm})}$ is a random $D_{k} \times D_{k}$ matrix in the $k$ th subsystem.

For a separable multipartite state $\rho$, there exists a decomposition, $W=\sqrt{\rho} U$, where each "subnormalized" pure state $\mid W_{i}>(i$ th column vector of $W)$ is a tensor product state in the form (9), thus is $O_{k \bar{k}}^{(\text {Semi-rdm) }}$-orthogonal to its complex conjugate. Similar to the above case, the matrix $W^{T} O_{k \bar{k}}^{(\text {Semi-rdm) }} W$ has to be a hollow matrix, which means the singular values $\left\{\lambda_{i}\right\}$ in decreasing order of the symmetric matrix,

$$
S=(\sqrt{\rho})^{T} O_{k \bar{k}}^{(\text {Semi-rdm) }} \sqrt{\rho},
$$

have to satisfy the condition (77). Now we conclude the separability criterion for a mixed multipartite state, that is, the condition (7) for the singular values of the above matrix (14) holds valid for all symmetric operators in the form 
(13). A violation by any operator in the form (13) is enough to declare the existence of multipartite entanglement. So every operator $O_{k \bar{k}}^{(\text {Semi-rdm) }}$ in the form (13) is a witness of multipartite entanglement under the violation of the condition (7). This huge class of entanglement witnesses can help us efficiently verify multipartite entanglement, even for mixed multipartite states. Here we also note that the singular values $\left\{\lambda_{i}\right\}$ of the symmetric matrix (14) are the square root of the eigenvalues of $S^{\dagger} S$, and as well the square root of the eigenvalues of the following matrix,

$$
\rho\left[O_{k \bar{k}}^{(\text {Semi-rdm })}\right]^{\dagger} \rho^{*} O_{k \bar{k}}^{(\text {Semi-rdm })}
$$

which is directly determined by the density matrix $\rho$ and an operator $O_{k \bar{k}}^{(\text {Semi-rdm) }}$ constructed by random matrices in the way (13).

To summarize, a group of symmetric operators with only two nonzero matrix elements are introduced to carry out the separability criterion of a general quantum state. Every symmetric operator, formulated as a tensor product of an antisymmetric operator in one subsystem and an antisymmetric operator in other subsystems as a whole, and their arbitrary linear combinations are entanglement witnesses for multipartite quantum states. Based on these symmetric operators, Wootters' separability criterion for two-qubit states is generalized to multipartite systems in arbitrary dimensions. How to distinguish all mixed entangled states from separable ones within a finite number of tests is the next challenging question worthy of investigation. Its answer might directly bring us a computable entanglement measure for mixed multipartite states.

This work was supported by the national Natural Science Foundation of China under Grant Nos. 11174118,11174026 and 11264018, and the Natural Science Foundation of Jiangxi Province, China under Grant No. $20114 B A B 212003$. We also thank Prof. J.P. Dowling for helpful discussions.

Note added.-After completion of this work, we became aware of a related work by Chen, Ma, Gühne, and Severini 22].

[1] A. Einstein, B. Podolsky, N. Rosen, Phys. Rev. 47(10), 777-780 (1935); E. Schrödinger, M. Born, Mathematical Proceedings of the Cambridge Philosophical Society 31(4), 555-563 (1935); E. Schrödinger; P. A. M. Dirac, Mathematical Proceedings of the Cambridge Philosophical Society 32(3), 446-452 (1936).

[2] R. F. Werner, Phys. Rev. A 40, 4277 (1989).

[3] C. H. Bennett, G. Brassard, S. Popescu, B. Schumacher, J. A. Smolin, and W. K. Wootters, Phys. Rev. Lett. 76, 722 (1996); C. H. Bennett, D. P. DiVincenzo, J. A. Smolin, and W. K. Wootters, Phys. Rev. A 54, 3824 (1996).

[4] V. Vedral, and M. B. Plenio, Phys. Rev. A 57, 1619 (1998).

[5] B. M. Terhal, and P. Horodecki, Phys. Rev. A 61, 040301 (2000); A. Sanpera, D. Bruß and M. Lewenstein, Phys. Rev. A 63, 050301 (2001).

[6] S. Hill, and W. K. Wootters, Phys. Rev. Lett. 78, 5022 (1997).

[7] W. K. Wootters, Phys. Rev. Lett. 80, 2245 (1998).

[8] K. Życzkowski, P. Horodecki, A. Sanpera, and M. Lewenstein, Phys. Rev. A 58, 883 (1998); G. Vidal, and R. F. Werner, Phys. Rev. A 65, 032314 (2002).

[9] R. Horodecki, P. Horodecki, M. Horodecki, K. Horodecki, Rev. Mod. Phys. 81, 865 (2009), and references therein.

[10] V. Coffman, J. Kundu, and W. K. Wootters, Phys. Rev. A 61, 052306 (2000).

[11] D. A. Meyer, and N. R. Wallach, e-print arXiv:quant-ph/ 0108104 (2001); J. Eisert, and H. J. Briegel, Phys. Rev. A 64, 022306 (2001); H. Barnum, and N. Linden, J. Phys. A 34, 6787 (2001).

[12] L. Amico, R. Fazio, A. Osterloh, and V. Vedral, Rev. Mod. Phys. 80, 517 (2008).

[13] A. Peres, Phys. Rev. Lett. 77, 1413 (1996).

[14] M. Horodecki, P. Horodecki, and R. Horodecki, Phys. Lett. A 223, 1 (1996).

[15] A. Uhlmann, Phys. Rev. A 62, 032307 (2000); A. Wong, and N. Christensen, Phys. Rev. A 63, 044301 (2001); F. Mintert, M. Kuś, and A. Buchleitner, Phys. Rev. Lett. 92, 167902 (2004).

[16] P. Rungta, V. Buzek, C. M. Caves, M. Hillery, and G. J. Milburn, Phys. Rev. A 64, 042315 (2001).

[17] F. Mintert, M. Kuś, and A. Buchleitner, Phys. Rev. Lett. 95, 260502 (2005); S. J. Akhtarshenas, J. Phys. A 38, 6777-6784 (2005).

[18] R. A. Horn and C. R. Johnson, Matrix Analysis (Cambridge University Press, New York, 1985), p. 205.

[19] B. M. Terhal, Phys. Lett. A 271, 319 (2000); M. Lewenstein, B. Kraus, J. I. Cirac, and P. Horodecki, Phys. Rev. A 62, 052310 (2000); B. M. Terhal, Linear Algebr. Appl. 323, 61 (2001); F. G. S. L. Brandão, Phys. Rev. A 72, 022310 (2005); G. Tóth, and O. Gühne, Phys. Rev. Lett. 94, 060501 (2005).

[20] V. Vedral, M. B. Plenio, M. A. Rippin, and P. L. Knight, Phys. Rev. Lett. 78, 2275 (1997).

[21] C. S. Yu, and H. S. Song, Phys. Rev. A 73, 022325 (2006).

[22] Z.H. Chen, Z.H. Ma, O. Gühne, and S. Severini, Phys. Rev. Lett. 109, 200503 (2012). 